\title{
A duopoly Logit model with price competition and strategic compatibility*
}

\author{
Nicolas Jonard ${ }^{\mathrm{a}, \mathrm{b}}$ Eric Schenk ${ }^{\mathrm{a}}$ \\ aBureau d'Economie Théorique et Appliquée (UMR 7522 CNRS) \\ 61, avenue de la Forêt Noire, 67000 Strasbourg, France \\ ${ }^{\mathrm{b}}$ Maastricht Economic Research Institute on Innovation and Technology \\ Tongersestraat 49, 6200 MD Maastricht, The Netherlands
}

March 30, 1999

\begin{abstract}
This paper provides an analysis of compatibility decisions with oligopolistic price setting in a duopoly logit model. We consider a sequential game in which firms first choose whether they supply compatible products and then set the price which is charged. The equilibrium compatibility configuration is the outcome of a trade-off between consumers valuation of compatibility and the loss of product differentiation which is associated with compatibility. Finally, it is shown that firms incentives towards compatibility tend to be socially insufficient.
\end{abstract}

Key words: compatibility, product differentiation, price competition, multinomial logit, welfare

JEL classification: L13, D4

\section{Introduction}

Product compatibility is a major issue in economies where agents communicate, exchange data or use common software. The fact that consumers valuate compatibility can be seen as the starting point of the literature on network externalities (see Katz and Shapiro, 1985 or the survey by Perrot, 1993). Besides their natural influence upon adoption (i.e. consumption) phenomena, network externalities should be considered as an essential matter for firms strategic behavior. For instance, the use of technical incompatibility by Microsoft has played a major role in the emergence and persistence

*Financial support from CNRS (programme 'Les enjeux économiques de l'innovation') is gratefully acknowledged. Authors e-mail adresses are n.jonard@merit.unimaas.nl and schenk@cournot.ustrasbg.fr 
of a dominant position in the market for operating system software (see Baseman et al., 1996). In the more recent 'Microsoft lawsuit', this firm has been alleged of introducing technical incompatibilities between its browser (Internet Explorer) and the Netscape browser.

In this paper, we put the emphasis on the interplay between compatibility and the intensity of price competition. On the one hand, firms are eager to supply compatible products since consumers make a higher valuation of such products (and so are more willing to buy them). Hence, compatibility enables producers to charge higher prices. On the other hand, we shall argue that consumers tend to perceive compatible products as closer substitutes. In turn, this may lead to increased price competition between firms.

The paper aims at giving an explicit representation of this trade-off in a sequential game in which firms first choose whether they sell compatible products and then set the price which is charged. Compatibility is achieved by affiliation to a common standard. The core assumption of the model is that compatibility is a determinant of product variety. In the case of network goods (i.e. goods yielding utility because they enable communication) some degrees of similarities between communication protocols and/or processing architectures are necessary in order to have compatibility (or interconnection) achieved. In the case of system goods, compatible hardware are able to use the same software products which leads to higher system similarity. In either case, consumers perceive compatible goods as being closer substitutes than incompatible ones. Accordingly, we consider in this paper that compatibility leads to a loss in product differentiation.

The model we propose here accounts for both increased substitutability via adhesion to a common standard and the fact that compatibility increases product utility. The paper is organized as follows. The model is outlined in section 2 . In section 3 , we characterize the subgame perfect equilibrium of the sequential game with strategic compatibility choice and then price competition. Finally, section 4 is devoted to an analysis of welfare.

\section{The model}

We first present the supply side and the concept of compatibility which is used, and then characterize demand under the various compatibility configurations.

\subsection{Firms and compatibility relationships}

Let us consider two monoproduct firms $(i=1,2)$ which first determine whether they sell compatible goods and then set the price of their products. Each good embodies a technical standard and compatibility is achieved by adoption of a common standard (see Economides and Flyer, 1998 for a similar approach). Conversely, products embodying distinct standards are assumed to be fully incompatible. 
Each firm is endowed with a specific (proprietary) standard. Firm 1 and firm 2 standards are labelled by $A$ and $B$ respectively. Standardization may occur through unilateral decision when a firm decides to adopt the other firm's standard, or it may be the outcome of a joint adoption procedure. In any case, the existence of a consensus can be considered as a requirement in order to have compatibility achieved (see e.g. Besen and Farrell, 1994 or Economides and Flyer, 1998). In the lack of a consensus, a firm which is willing to be incompatible is either able to protect access to its standard, or to introduce ex post incompatibilities. Accordingly, compatibility is achieved if and only if both firms wish to be compatible. In this case, we shall assume that they both agree on adopting standard $A^{1}$. Conversely, incompatibility prevails as soon as one firm is not willing to be compatible.

\subsection{Demand}

We consider a population of $N$ consumers $(N \gg 2)$ with the following discrete choice sequence. In the first stage, each consumer chooses one of the available standards or an outside (or no-purchase) alternative indexed by 0 . This alternative accounts for non-zero demand elasticity in response to price changes (see Anderson and de Palma, 1992). The utility level associated with the outside alternative is given exogenously and conditions the volume of demand addressed to the firms. In the second step, consumers who have decided to buy something choose a good which embodies the technical standard adopted in the first stage. The first step of consumers decision can be thought of as a "network choice", while the second one corresponds to a "product choice".

One has to distinguish the case in which goods are compatible from that of incompatible products. When incompatibility prevails, each standard is represented by a single good on the market. Then, the second step of consumers choice is trivial. When firms sell compatible products, they both embody standard $A$. Products which embody a common standard are referred to as variants of this standards. Then, conditionally to the fact that he has decided to buy something, each consumer chooses his most preferred variant in the second step.

Both the competing standards and the variants of a same standard are assumed to be horizontally differentiated. We adopt a structure that is widely used in quantitative marketing, empirical industrial economics and the theory of product differentiation, namely the multinomial logit (MNL in the sequel, see Anderson et al., 1992). Overall demand is therefore represented by a nested MNL (Ben-Akiva, 1973). Since compatible products share many features in common, we consider that they are less differentiated than incompatible ones. An equivalent statement is that the dispersion of consumers preferences across standards is larger that consumer heterogeneity at the variant level. Figure 1 gives a symbolic representation of the demand structure in the two configurations.

Having described the decision sequence of consumers, we turn to the formal definition of demand in each configuration.

\footnotetext{
${ }^{1}$ We thereby omit the potential coordination problem which may arise from firms standard choice.
} 


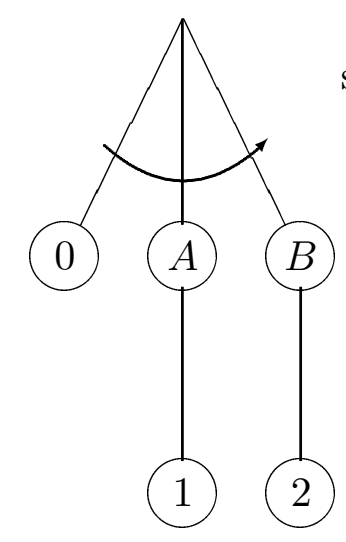

(i)

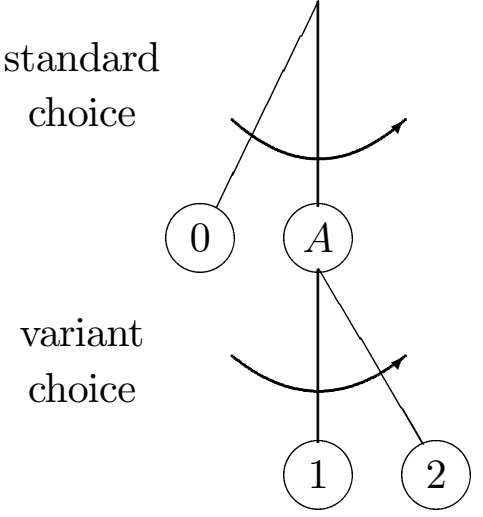

(ii)

Figure 1: The demand structure in the case of incompatible goods $(i)$ and in the case of compatible variants $(i i)$.

\subsubsection{Demand when products are incompatible}

In our model, goods are incompatible if and only if each firm adopts its specific standard. Then, the utility of a standard and the utility of the corresponding good coincide.

Horizontal product differentiation means that consumers do not agree on their natural preferences for the competing products. We consider that a consumer chosen at random from the population of $N$ consumers has a conditional indirect utility function

$$
\widetilde{u}_{i}=R-p_{i}+\varepsilon_{i}, \text { for } i=1,2 .
$$

Parameter $R$ stands for the consumer's income (which is assumed to be large). The $\varepsilon_{i}$ s represent consumers idiosyncratic tastes about the goods, and they are i.i. double exponentially distributed with parameter $\mu$. The outside alternative has an associated utility given by

$$
\widetilde{u}_{0}=R+u_{0}+\varepsilon_{0}
$$

where $\varepsilon_{0}$ is i.i. double exponentially distributed along with $\varepsilon_{1}$ and $\varepsilon_{2}$. The purchase probability for product $i$ writes

$$
\mathbf{P}_{i}=\frac{\exp \left(-p_{i} / \mu\right)}{\sum_{j=1,2} \exp \left(-p_{j} / \mu\right)+\exp \left(u_{0} / \mu\right)}, \text { for } i=1,2 .
$$

Note that the case $u_{0} \rightarrow-\infty$ corresponds to the standard MNL in which overall demand is inelastic. Finally, expected demand addressed to firm $i(i=1,2)$ is given by $N \mathbf{P}_{i}$. 


\subsubsection{Demand when products are compatible}

When compatibility prevails, standard $A$ is represented by two products on the market, while standard $B$ is not available. Consumers attach a common positive value $\phi$ to product compatibility, which captures their expectations about positive effects -such as network externalities- induced by compatibility ${ }^{2}$. An alternative interpretation of $\phi$ is that adoption of a common standard by firms prevents consumers from buying costly converters in order to achieve compatibility (see e.g. Farrell and Saloner, 1992). Hence $\phi$ represents the amount of money which is saved when converters become useless. Note that we consider compatibility to be valuable per se, and do not explicitly model how network externalities alter utility. This allows for an alleviated treatment of compatibility choice by avoiding the complications that would result from considering a system of implicit equations (Katz and Shapiro, 1985; de Palma and Leruth, 1993).

Variants of standard $A$ are assumed to be horizontally differentiated. The conditional indirect utility function of a consumer chosen at random from the population of those who decided to buy something is given by

$$
\widetilde{u}_{i}=R+\phi-p_{i}+\varepsilon_{i}^{\prime}, \text { for } i=1,2,
$$

where the $\varepsilon_{i}^{\prime} \mathrm{s}$ again represent consumers idiosyncratic tastes for the variants and are i.i. double exponentially distributed with parameter $\mu_{C}$. The assumption that compatibility increases product substitutability writes $\mu_{C}<\mu$. The purchase probability for compatible product $i$, conditional to the decision of purchasing anything at all, is given by

$$
\mathbf{P}_{i / A}=\frac{\exp \left(-p_{i} / \mu_{C}\right)}{\sum_{j=1,2} \exp \left(-p_{j} / \mu_{C}\right)}, \text { for } i=1,2 .
$$

It should be clear that the two compatible goods do not directly compete with the outside alternative. Following Ben-Akiva and Lerman (1979), the attractiveness of standard $A$ (which stands for the pool of compatible goods) is now measured by the expected value of the maximum utility derived within that pool, according to

$$
u_{A}=\mu_{C} \ln \sum_{j=1,2} \exp \left[\left(\phi-p_{j}\right) / \mu_{C}\right] .
$$

This index reflects the fact that full information about variants is only revealed when the consumer has decided to buy something. In the first step of the decision procedure, a consumer chosen at random from the total population of consumers has a conditional indirect utility of adopting standard $A$ writing

$$
\widetilde{u}_{A}=u_{A}+\varepsilon_{A}
$$

\footnotetext{
${ }^{2}$ These may be direct when compatibility enables communication between agents, or indirect when compatibility makes it possible for different hardware products to use common software goods (see Katz and Shapiro, 1985 or the survey by Perrot, 1993). If we were to consider $\phi<0$, compatibility would be associated to (negative) congestion effects.
} 
where $\varepsilon_{A}$ is i.i. double exponentially distributed along with $\varepsilon_{0}$. The purchase probability for standard $A$ is now given by

$$
\mathbf{P}_{A}=\frac{\exp \left(u_{A} / \mu\right)}{\exp \left(u_{A} / \mu\right)+\exp \left(u_{0} / \mu\right)} .
$$

Finally, expected demand addressed to firm $i(i=1,2)$ writes $N \mathbf{P}_{i, A}=N \mathbf{P}_{A} \mathbf{P}_{i / A}$.

Having defined demand in each configuration, we now seek the subgame perfect Nash equilibrium of the compatibility-then-price game.

\section{The equilibrium}

We seek a subgame perfect Nash equilibrium in which firms first choose whether to be compatible and then set prices. The compatibility strategy of firm $i(i=1,2)$ is defined by $s_{i} \in\{I, C\}$, where $C(I)$ stands for compatibility (incompatibility). Given any compatibility configuration $s \equiv\left(s_{1}, s_{2}\right)$ determined in the first stage, the equilibrium of the price subgame is given by $p_{1}^{*}(s)$ and $p_{2}^{*}(s)$ such that

$$
\pi_{i}\left(p_{i}^{*}, p_{j}^{*} ; s\right) \geq \pi_{i}\left(p_{i}, p_{j}^{*} ; s\right) \text {, for all } p_{i} \geq 0, i=1,2 \text { and } j \neq i .
$$

Denote the profit functions evaluated at the second stage equilibrium $p^{*}(s)$ by $\hat{\pi}_{i}(s) \equiv$ $\pi_{i}\left[p^{*}(s) ; s\right]$. The equilibrium of the compatibility game is then given by $s_{1}^{*}$ and $s_{2}^{*}$ satisfying

$$
\hat{\pi}_{i}\left(s_{i}^{*}, s_{j}^{*}\right) \geq \hat{\pi}_{i}\left(s_{i}, s_{j}^{*}\right), \text { for all } s_{i} \in\{I, C\}, i=1,2 \text { and } j \neq i .
$$

A subgame perfect Nash equilibrium for the compatibility-then-price game is defined by $s^{*}$ and $p^{*}(s)$ for all compatibility configurations $s$. The corresponding equilibrium path writes $s^{*}$ and $p^{*}\left(s^{*}\right)$.

We proceed by backward induction, defining the price equilibrium and then the equilibrium of the compatibility subgame.

\subsection{The price equilibrium}

Let us recall that compatibility actually prevails if and only if both firms wish to be compatible. Accordingly, one only needs to analyze the overall incompatibility and the compatibility cases in order to account for the four possible configurations of the compatibility strategies.

\subsubsection{The price subgame for incompatible goods}

Firms are assumed to produce at constant marginal cost $c$, so that firm $i$ 's profit is $\pi_{i}=\left(p_{i}-c\right) N \mathbf{P}_{i}$. An important derivative property of relation (3) is that $\partial \mathbf{P}_{i} / \partial p_{i}=$ $-\mathbf{P}_{i}\left(1-\mathbf{P}_{i}\right) / \mu$ for $i=1,2$. Therefore, the profit derivative for firm $i$ is

$$
\frac{\partial \pi_{i}}{\partial p_{i}}=-N\left(p_{i}-c\right) \frac{\mathbf{P}_{i}\left(1-\mathbf{P}_{i}\right)}{\mu}+N \mathbf{P}_{i}, \text { for } i=1,2,
$$


with second derivative

$$
\frac{\partial^{2} \pi_{i}}{\partial p_{i}^{2}}=-N\left(p_{i}-c\right) \frac{\mathbf{P}_{i}\left(1-\mathbf{P}_{i}\right)\left(2 \mathbf{P}_{i}-1\right)}{\mu^{2}}-2 N \frac{\mathbf{P}_{i}\left(1-\mathbf{P}_{i}\right)}{\mu}
$$

Evaluating (12) at any point where (11) is equal to zero gives $-N \mathbf{P}_{i} / \mu$, indicating that the profit function of each firm is strictly concave in its own price. Therefore firm $i^{\prime}$ s best reply $p_{i}^{b r}\left(p_{j}\right)$ is uniquely defined. Moreover, uniqueness of the equilibrium is ensured if the best reply functions satisfy $\partial p_{i}^{b r} / \partial p_{j}<1$ for $i=1,2$ and $j \neq i$ (see e.g. Anderson et al., 1992). One can check that this inequality is satisfied, leading to the following proposition.

Proposition 1 (Anderson and de Palma, 1992) When goods are incompatible and when demand is defined by relation (3), the unique equilibrium price is implicitly given by

$$
p_{i}^{*}=p_{I}=c+\frac{\mu}{1-\mathbf{P}_{I}},
$$

with

$$
\mathbf{P}_{i}=\mathbf{P}_{I}=\left[2+\exp \left(\frac{u_{0}+p_{I}}{\mu}\right)\right]^{-1}, i=1,2 .
$$

Individual profits in this configuration are denoted by $\pi_{I}$. The equilibrium price and output per firm are decreasing in the relative attractiveness of the outside alternative $u_{0}$. Moreover, prices and profits rise with $\mu$ (other results can be found in Anderson et de Palma, 1992).

\subsubsection{The price subgame for compatible goods}

When firms sell compatible products, firm $i$ 's profit writes $\pi_{i}=\left(p_{i}-c\right) N \mathbf{P}_{i, A}$. The first order condition for profit maximization of firm $i$ becomes

$$
\frac{\partial \mathbf{P}_{i, A}}{\partial p_{i}}\left(p_{i}-c\right)+\mathbf{P}_{i, A}=0, \text { for } i=1,2 .
$$

Since $\mathbf{P}_{i, A}=\mathbf{P}_{A} \mathbf{P}_{i / A}(i=1,2)$, one has

$$
\frac{\partial \mathbf{P}_{i, A}}{\partial p_{i}}=\frac{\partial \mathbf{P}_{A}}{\partial p_{i}} \mathbf{P}_{i / A}+\mathbf{P}_{A} \frac{\partial \mathbf{P}_{i / A}}{\partial p_{i}}
$$

The first term reflects the impact of price changes on the demand volume addressed to standard $A$, whereas the second term stands for inter-firm competition. These are respectively

$$
\frac{\partial \mathbf{P}_{A}}{\partial p_{i}}=\frac{\partial \mathbf{P}_{A}}{\partial u_{A}} \frac{\partial u_{A}}{\partial p_{i}}=-\frac{\mathbf{P}_{A}\left(1-\mathbf{P}_{A}\right)}{\mu} \mathbf{P}_{i / A},
$$

and

$$
\frac{\partial \mathbf{P}_{i / A}}{\partial p_{i}}=-\frac{\mathbf{P}_{i / A}\left(1-\mathbf{P}_{i / A}\right)}{\mu_{C}} .
$$


Evaluating the second derivative of firm $i$ 's profit function when the first order condition is satisfied yields

$$
\begin{aligned}
\frac{\partial^{2} \pi_{i}}{\partial p_{i}^{2}}= & -\frac{\mathbf{P}_{A} \mathbf{P}_{i / A}}{\mu \mu_{C}} . \\
& \frac{\mu^{2}\left(1-\mathbf{P}_{i / A}\right)+\mu_{C}\left(1-\mathbf{P}_{A}\right) \mathbf{P}_{i / A}\left[\mu_{C} \mathbf{P}_{i / A}+\mu\left(1-\mathbf{P}_{i / A}\right)\right]}{\mu_{C}\left(1-\mathbf{P}_{A}\right) \mathbf{P}_{i / A}+\mu\left(1-\mathbf{P}_{i / A}\right)}
\end{aligned}
$$

which is negative. Hence, the profit function of each firm is concave in its own price and a symmetric equilibrium exists. Again, it is checked that the equilibrium is unique by showing that the best reply functions satisfy $\partial p_{i}^{b r} / \partial p_{j}<1$ for $i=1,2$ and $j \neq i$. Therefore, the equilibrium with compatible products is characterized by the following proposition.

Proposition 2 When goods are compatible and demand is defined by relations (5) and (8), the unique equilibrium price is given by

$$
p_{i}^{*}=p_{C}=c+\frac{\mu}{\left(\mu_{C}+\mu\right) / 2 \mu_{C}-\mathbf{P}_{C}}
$$

with

$$
\mathbf{P}_{i}=\mathbf{P}_{C}=\left[2+2 \exp \left(\frac{u_{0}-\phi-\mu_{C} \ln 2+p_{C}}{\mu}\right)\right]^{-1}, i=1,2 .
$$

Proof Since firms are symmetric at equilibrium, we have $\mathbf{P}_{i / A}=1 / 2$. Hence, relation (16) becomes

$$
\frac{\partial \mathbf{P}_{i, A}}{\partial p_{i}}=-\frac{\mathbf{P}_{A}}{4}\left[\frac{1-\mathbf{P}_{A}}{\mu}+\frac{1}{\mu_{C}}\right] .
$$

Substituting in the first order condition (15) gives an optimal price of

$$
p_{C}=c+\frac{2}{\frac{1-\mathbf{P}_{A}}{\mu}+\frac{1}{\mu_{C}}} .
$$

Since we have $\mathbf{P}_{A}=2 \mathbf{P}_{C}$, we can write

$$
\begin{aligned}
\mathbf{P}_{C} & =\left[2+2 \exp \left(\frac{u_{0}-u_{A}}{\mu}\right)\right]^{-1} \\
& =\left[2+2 \exp \left(\frac{u_{0}-\phi-\mu_{C} \ln 2+p_{C}}{\mu}\right)\right]^{-1},
\end{aligned}
$$

since $u_{A}=\mu_{C} \ln \sum_{j=1,2} \exp \left[\left(\phi-p_{j}\right) / \mu_{C}\right]=\mu_{C} \ln 2+\phi-p_{C}$.

When compatibility prevails, individual profits at equilibrium are denoted $\pi_{C}$. One can notice that if $\mu_{C}=\mu$, equilibrium prices in the compatibility and incompatibility cases are the same, except profits for compatible firms include the term $\phi$. Having defined equilibrium prices, we are able to analyze the first stage of the sequential game. 


\subsection{The compatibility game}

We now investigate the outcome of the compatibility game given optimal decisions in the second stage of the game. The structure of the model is such that firms face an arbitrage between product differentiation and consumers willingness to pay for the goods (i.e. product "quality").

According to equilibrium condition (10), firm $i(i=1,2)$ chooses compatibility at the subgame perfect Nash equilibrium if and only if $\hat{\pi}_{i}\left(C, s_{j}^{*}\right) \geq \hat{\pi}_{i}\left(I, s_{j}^{*}\right)$ for $j \neq i$. Moreover, since the game is symmetric, there are no conflicts between firms incentives towards compatibility ${ }^{3}$. Hence, both firms choose compatibility at equilibrium if and only if compatibility yields the highest individual profits, i.e. $\pi_{C} \geq \pi_{I}$.

\subsubsection{Markup versus quantity in the MNL}

To begin with, let us consider the situation where there are no expected benefits from compatibility, i.e. $\phi=0$. In this case we are interested in analyzing firms tradeoff between charging high prices or selling high quantities. To put it differently, we seek to know whether firms might strategically switch to a market in which product differentiation is lower in order to attract a higher volume of demand.

We shall refer to this as the differentiation-then-price game. It involves the comparison of $\pi_{C}$ and $\pi_{I}$, and we get the following proposition.

Proposition 3 When demand is described by a nested $M N L$, the unique equilibrium of the differentiation-then-price game implies firms choosing maximal differentiation.

Proof See appendix A.

The situation is illustrated by the solid curve in figure 2. The profits of compatible producers rise with the level of product differentiation, starting from zero when goods are perfect substitutes $\left(\mu_{C}=0\right)$ and competition bids prices down to the marginal cost.

Proposition 3 strengthens the well known maximal differentiation result (see e.g. d'Aspremont et al., 1979), according to which firms differentiate their products in order to relax competition. In our model, firms wish to differentiate their products even though high prices have a negative influence upon the volume of demand addressed to the firms. We now turn to the case where consumers attach a strictly positive value to product compatibility.

\subsubsection{The trade-off between compatibility and differentiation}

When consumers have a positive valuation of compatibility $(\phi>0)$, the compatibilitythen-price game has a less immediate outcome. The dotted curve in figure 2 depicts firms profits as $\mu_{C}$ is varied, for non-zero $\phi$. Compatibility now has a positive influence on consumers willingness to pay for the goods. Hence, equilibrium profits for

\footnotetext{
${ }^{3}$ Contradictory incentives can be found in de Palma and Leruth (1996) or Economides and Flyer (1998) in a vertical differentiation framework.
} 


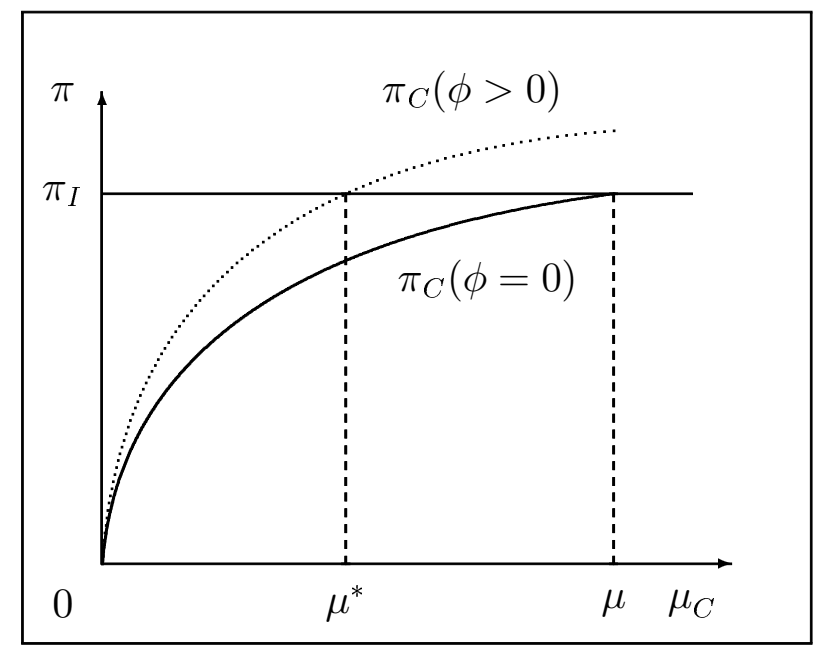

Figure 2: Profits for compatible firms as functions of $\mu_{C}$ with (dotted curve) and without (solid curve) valuation of compatibility

compatible producers are higher than in the previous case for any $\mu_{C} \geq 0$, leading to the following result.

Proposition 4 For any positive value of $\phi$, there exists a threshold value of $\mu_{C}$, denoted $\mu^{*}$, such that the subgame perfect Nash equilibrium of the compatibility-thenprice game entails incompatibility when $\mu_{C}<\mu^{*}$ and compatibility when $\mu_{C}>\mu^{*}$. Formally, $s_{1}^{*}=s_{2}^{*}=I$ when $\mu_{C}<\mu^{*}$ and $s_{1}^{*}=s_{2}^{*}=C$ when $\mu_{C}>\mu^{*}$.

Proof It suffices to note that for any positive $\phi$, profits $\pi_{C}$ monotonically increase with $\mu_{C}$ (see appendix A), up to a value that strictly exceeds $\pi_{I}$ when $\mu_{C}=\mu$. Hence, there is a unique value of $\mu_{C}$, denoted $\mu^{*}$ (with $\mu^{*}<\mu$ ), such that $\pi_{I}=\pi_{C}$.

An immediate corollary of this proposition is that for any couple $\left(\mu, \mu_{C}\right)$ satisfying $\mu_{C}<\mu$, there exists a finite value of $\phi$ (denoted $\phi^{*}$ ) above which compatibility is observed at the unique subgame perfect Nash equilibrium.

The model shows that standardization is achieved provided the valuation of compatibility by consumers dominates the increased competition which is associated with a lower product differentiation. Conversely, full incompatibility occurs when the loss of horizontal differentiation is too high with regard to consumers valuation of compatibility. As extreme cases, the absence of expected compatibility benefits would induce all firms to supply incompatible standards which are more differentiated, whereas strong substitutability among standards leads to overall compatibility.

\subsection{Welfare analysis}

The MNL offers a convenient framework for welfare analysis. According to Ben-Akiva and Lerman (1979), consumers surplus writes

$$
S=N \mu \ln \sum_{k \in K} \exp \left(\frac{u_{k}}{\mu}\right),
$$


where

$$
K=\left\{\begin{array}{l}
\{0, A, B\} \text { if goods are incompatible } \\
\{0, A\} \text { if goods are compatible }
\end{array}\right.
$$

Evaluating welfare at the symmetric equilibrium when incompatibility prevails yields

$$
W_{I}=N \mu \ln \left[2 \exp \left(-\frac{p_{I}}{\mu}\right)+\exp \left(\frac{u_{0}}{\mu}\right)\right]+2\left(p_{I}-c\right) N \mathbf{P}_{I}
$$

where the first term stands for consumers surplus and the second one for firms profits. Similarly, welfare at the symmetric equilibrium when compatibility prevails writes

$$
W_{C}=N \mu \ln \left[\exp \left(\frac{\mu_{C} \ln 2+\phi-p_{C}}{\mu}\right)+\exp \left(\frac{u_{0}}{\mu}\right)\right]+2\left(p_{C}-c\right) N \mathbf{P}_{C} .
$$

Product compatibility has a indirect influence on consumers surplus, via the price which is charged. Moreover, compatibility has two direct effects on consumers surplus. On the one hand, consumers attach some value to product compatibility. On the other hand, consumers are affected by the degree of product differentiation which prevails when compatibility is achieved. Indeed, the MNL demand structure captures consumers 'taste for diversity' (see Anderson et al., 1992). Increasing product similarity via compatibility (i.e. decreasing the value of $\mu_{C}$ ) therefore has a negative influence on consumers surplus.

The price equilibrium is implicitly defined and analytical comparison of $W_{I}$ and $W_{C}$ is difficult. Yet, the following numerical exercise illustrates the potential inefficiency of the equilibrium outcome. Setting $u_{0}=0, \mu=10$ and $N=100$, we vary those parameters which define the private incentives towards compatibility, namely

\begin{tabular}{|c|c|c|c|c|c|c|c|c|}
\hline \multirow{3}{*}{$\mu_{C}$} & \multicolumn{8}{|c|}{ Compatibility valuation } \\
\hline & \multicolumn{2}{|c|}{$\phi=0$} & \multicolumn{2}{|c|}{$\phi=1$} & \multicolumn{2}{|c|}{$\phi=5$} & \multicolumn{2}{|c|}{$\phi=10$} \\
\hline & $\overline{W_{C} / W_{I}}$ & $\pi_{C} / \pi_{I}$ & $\overline{W_{C} / W_{I}}$ & $\overline{\pi_{C} / \pi_{I}}$ & $\overline{W_{C} / W_{I}}$ & $\overline{\pi_{C} / \pi_{I}}$ & $\overline{W_{C} / W_{I}}$ & $\overline{\pi_{C} / \pi_{I}}$ \\
\hline 10 & 1.00 & 1.00 & 1.56 & 1.07 & 1.66 & 1.36 & 1.81 & 1.77 \\
\hline 9 & 0.97 & 0.94 & 1.54 & 1.00 & 1.63 & 1.28 & 1.77 & 1.67 \\
\hline 8 & 0.95 & 0.88 & 1.51 & 0.94 & 1.61 & 1.20 & 1.74 & 1.56 \\
\hline 7 & 0.93 & 0.82 & 1.49 & 0.87 & 1.58 & 1.11 & 1.71 & 1.44 \\
\hline 6 & 0.90 & 0.75 & 1.48 & 0.80 & 1.56 & 1.01 & 1.67 & 1.31 \\
\hline 5 & 0.88 & 0.67 & 1.47 & 0.72 & 1.54 & 0.90 & 1.64 & 1.16 \\
\hline 4 & 0.86 & 0.58 & 1.46 & 0.62 & 1.52 & 0.78 & 1.61 & 0.99 \\
\hline 3 & 0.84 & 0.48 & 1.45 & 0.51 & 1.50 & 0.63 & 1.57 & 0.79 \\
\hline 2 & 0.82 & 0.35 & 1.45 & 0.37 & 1.49 & 0.46 & 1.53 & 0.57 \\
\hline 1 & 0.79 & 0.20 & 1.44 & 0.21 & 1.46 & 0.25 & 1.49 & 0.30 \\
\hline
\end{tabular}
$\mu_{C}$ and $\phi$. Table 1 summarizes the equilibrium and socially optimal welfares obtained with $\phi=0,1,5,10$ and $\mu_{C}=1, \ldots, 10$.

Table 1: Welfare analysis

In each case, the first column depicts the ratio $W_{C} / W_{I}$ of the welfare when compatibility is achieved over the welfare associated with overall incompatibility, whereas 
the second column depicts the ratio $\pi_{C} / \pi_{I}$ of firms profits in both configurations ${ }^{4}$. The reference values are $W_{I} \simeq 915,03$ and $\pi_{I} \simeq 453,51$. Compatibility is observed at the subgame perfect Nash equilibrium of the compatibility-price game if and only if $\pi_{C} / \pi_{I}>1$. Hence, the equilibrium compatibility configuration is socially optimal if and only if $\pi_{C} / \pi_{I}$ and $W_{C} / W_{I}$ are both either larger or smaller than one. Clearly, this is not always the case and the following proposition holds.

Proposition 5 The equilibrium outcome might be socially suboptimal provided consumers have a non-zero valuation of compatibility.

When consumers do not valuate compatibility $(\phi=0)$, the equilibrium and the social optimum coincide and they entail maximal differentiation (i.e. incompatibility). By contrast, as soon as the value attached to compatibility is non-zero, there may appear a dissonance between private and public incentives towards compatibility. The amount of product differentiation $\mu_{C}$ corresponding to the situation where $\pi_{C} / \pi_{I}=1$ (bold numbers) is the threshold value $\mu^{*}$ (defined in proposition 4) below which compatibility is not achieved at equilibrium. Furthermore, the welfare ratio indicates that compatibility is always benefic from a social point of view when $\phi>0$. Hence, the outcome of the compatibility-then-price game is socially suboptimal as soon as $\phi>0$ and $0 \leq \mu_{C}<\mu^{*}$.

Firms incentives towards compatibility tend to be too low when standardization entails high product similarity. According to table 1, the higher consumers valuation of compatibility, the lower the threshold value of $\mu_{C}$ above which compatibility is achieved. Hence firms strategic interaction is "more likely" to lead to an inefficient outcome when consumers valuation of compatibility is low.

\section{Conclusion}

By means of a nested multinomial demand structure, we have modelled how compatibility simultaneously increases consumers valuation of compatible goods and product substitutability. Within a sequential game, we have analyzed the interplay between firms compatibility decisions and the intensity of price competition. First, it was shown that maximal differentiation emerges at equilibrium when consumers do not attach any value to product compatibility. Actually, demand elasticity is non-zero and firms are willing to differentiate their products even though high prices have a negative impact on the volume of demand. This result no longer holds when consumers have a strictly positive valuation of compatibility. In this case, firms compatibility decisions are the outcome of a trade-off between higher product "quality" and fierce price competition. In turns out that compatibility is achieved at equilibrium provided the amount of product differentiation among compatible good remains above a threshold value.

\footnotetext{
${ }^{4}$ Detailed analysis shows that the value of $\phi$ exerts a positive influence upon social welfare, firms profits and consumers surplus. Moreover, increasing product substitutability (diminishing $\mu_{C}$ ) rises consumers surplus and social welfare, but decreases individual profits.
} 
Even though the model does not yield explicit solutions to the price subgame, some insights into the welfare implications of compatibility decisions could be given. Especially, we have shown that the equilibrium outcome is socially suboptimal when consumers valuate compatibility and when the latter entails a high loss in product differentiation. While welfare maximization would call for product compatibility, firms prefer to make their products incompatible in order to prevent price competition from being too tough.

\section{A Proof of proposition 3}

Equations (20) and (21) yield

$$
\pi_{C}=\left(p_{C}-c\right) N \mathbf{P}_{C}=\frac{\mu N \mathbf{P}_{C}}{\left(\mu_{C}+\mu\right) / 2 \mu_{C}-\mathbf{P}_{C}} .
$$

Differentiating w.r.t. $\mu_{C}$ gives

$$
\frac{\partial \pi_{C}}{\partial \mu_{C}}=2 \mu N \frac{\mu_{C}\left[\partial \mathbf{P}_{C} / \partial \mu_{C}\right]\left(\mu_{C}+\mu\right)+\mu \mathbf{P}_{C}}{\left(\mu_{C}+\mu-2 \mathbf{P}_{C} \mu_{C}\right)^{2}} .
$$

Using the implicit functions theorem, we show that

$$
\frac{\partial \mathbf{P}_{C}}{\partial \mu_{C}}=\frac{\mathbf{P}_{C}\left(1-2 \mathbf{P}_{C}\right)\left(2 \mu^{2}-\ln 2 \cdot\left(\mu_{C}+\mu-2 \mathbf{P}_{C} \mu_{C}\right)^{2}\right)}{\mu\left(-\mu^{2}-2 \mu \mu_{C}+4 \mu \mu_{C} \mathbf{P}_{C}-\mu_{C}^{2}+4 \mathbf{P}_{C}^{2} \mu_{C}^{2}\right)} .
$$

After some manipulation, we obtain

$$
\frac{\partial \pi_{C}}{\partial \mu_{C}}=2 N \mathbf{P}_{C} \frac{\ln 2 \cdot \mu_{C}\left(1-2 \mathbf{P}_{C}\right)\left(\mu+\mu_{C}\right)\left[\mu+\mu_{C}\left(1-2 \mathbf{P}_{C}\right)\right]+\mu^{2}\left[\mu-\mu_{C}\left(1-2 \mathbf{P}_{C}\right)\right]}{\left[\left(\mu+\mu_{C}\right)^{2}-4 \mu_{C} \mathbf{P}_{C}\left(\mu_{C} \mathbf{P}_{C}+\mu\right)\right] \cdot\left[\mu+\mu_{C}\left(1-2 \mathbf{P}_{C}\right)\right]} .
$$

Clearly all terms at the numerator are positive since $\mathbf{P}_{C}<1 / 2$ (in the most favorable case $u_{0} \rightarrow-\infty$, and the two firms attract the whole demand, i.e. $2 \mathbf{P}_{C} \rightarrow 1$ ). This also entails that $\left(\mu+\mu_{C}\right)^{2}-4 \mu_{C} \mathbf{P}_{C}\left(\mu_{C} \mathbf{P}_{C}+\mu\right)$ is larger than the same expression at $\mathbf{P}_{C}=1 / 2$, which is $\mu^{2}>0$. Hence $\partial \pi_{C} / \partial \mu_{C}>0$ for any $\mu_{C}$ and so profits monotonically rise with $\mu_{C}$, being eventually equal to $\pi_{I}$ when $\mu_{C}=\mu$. Therefore, maximal differentiation is the unique equilibrium for the differentiation-price game.

\section{References}

Anderson S.P., de Palma A. and Thisse J.-F., (1992), Discrete Choice Theory of Product Differentiation, MIT Press, Cambridge.

Anderson S.P. and de Palma A., (1992), "The logit as a model of product differentiation", Oxford Economic Papers, 44, 51-67.

Baseman K.C., Warren-Boulton F.R. and Woroch G.A., (1996), "Exclusionary behavior in a market for operating system software: the case of Microsoft", mimeo. 
Ben-Akiva M., (1973), Structure of Passenger Travel Demand Models, Ph.D. dissertation, MIT.

Ben-Akiva M. and Lerman S., (1979), "Dissagregate travel and mobility choice models and measures of accessibility" in Hensher D.A. and Stopher P.R. (eds.), Behavioral Travel Modelling, London: Croon Helm.

Besen S.M. and Farrell J., (1994), "Choosing how to compete : strategies and tactics in standardization", Journal of Economic Perspectives, 8, 117-131.

d'Aspremont C., Gabszewicz J.J. and Thisse J.-F., (1979), "On Hotelling's 'stability in competition'", Econometrica, 47, 1145-1150.

de Palma A. and Leruth L., (1993), "Equilibrium in competing networks with differentiated products", Transportation Science, 27, 73-80.

de Palma A. and Leruth L., (1996), "Variable willingness to pay for network externalities with strategic standardization decisions", European Journal of Political Economy, 12, 235-251.

Economides N. and Flyer F., (1998), "Equilibrium coalition structures in markets for network goods", Annales d'économie et de statistique, 49/50, 361-380.

Farrell J. and Saloner G., (1992), "Converters, compatibility, and the control of interfaces", Journal of Industrial Economics, 40, 9-35.

Katz M. and Shapiro C., (1985), "Network externalities, competition and compatibility", American Economic Review, 75, 424-440.

Perrot A., (1993), "Compatibility, networks and competition: a review of recent advances", Transportation Science, 27, 62-72. 